\title{
Isolated Activation of the Infraspinatus Muscle in Four Manual Muscle Testing Positions
}

\author{
In-cheol Jeon, PT, Ph.D ${ }^{1}$; Sung-min Ha, PT, Ph.D²; Sung-hoon Jung, PT, Ph.D ${ }^{3}$ \\ ${ }^{1}$ Department of Physical Therapy, College of Life and Health Science, Hoseo University, South Korea \\ ${ }^{2}$ Department of Physical Therapy, College of Health Science, Sangji University, South Korea \\ ${ }^{3}$ Department of Physical Therapy, College of Health Science, Yonsei University, South Korea
}

Background To normalize infraspinatus muscle activity among different exercises or positions, it is necessary to isolate the infraspinatus from the surrounding musculature.

Purpose We sought to determine the best position in which to obtain an isolated infraspinatus electromyography (EMG) signal for normalization of the infraspinatus muscle in four selected positions.

Study design Comparative, repeated measures design.

Methods To measure infraspinatus maximal voluntary muscle contraction (MVIC), 13 healthy participants performed four test positions (side-lying with the shoulder adducted, prone, shoulder abducted, and side-lying wiper external rotation [SWE]). EMG activity was measured in the infraspinatus, middle trapezius, and posterior deltoid muscles.

Results The EMG ratio was significantly higher for SWE than for the other test positions. To maximize the EMG ratio, high infraspinatus activity and low surrounding muscle (middle trapezius and posterior deltoid) activity are required.

Conclusions We recommend switching from prone external rotation to SWE to normalize the infraspinatus EMG signal.

Key words Electromyography; Infraspinatus; Normalization; Side-lying wiper external rotation.

JMST

2018; 2(2): 38-42

Published Online Dec 31, 2018

pISSN 2635-8573

eISSN 2635-8581

\section{Article History}

Received 02 October 2018

Revised 02 October 2018

(1st)

Revised 01 November 2018

(2nd)

Accepted 13 November 2018

\section{CONTACT}

hsm98@sangji.ac.kr

Sung-min Ha,

Department of Physical

Therapy, College of

Health Science, Sangji

University, South Korea

\section{This is an Open-Access article distributed under the terms of the Creative Commons Att- ribution Non-Commercial Li- cense (http://creativecommons.
org/licenses/by-nc/4.0) which permits unrestricted non-co- mmercial use, distribution, and reproduction in any me- dium, provided the original} work is properly cited.

\section{INTRODUCTION}

The most common way to generate a reference level for muscle activation to normalize infraspinatus electromyography (EMG) data is to record the maximum voluntary isometric contraction (MVIC) to identify the effects of exercise or intervention on infraspinatus muscle strengthening $^{1-3}$. Unlike other normalization methods, including subMVIC and reference voluntary contraction (RVC), MVIC is advantageous because it has physiological meaning; derived data are expressed relative to the maximum ${ }^{4}$.

For infraspinatus normalization, clinicians or researchers commonly take measurements while the subject is in the prone position ${ }^{5-8}$. However, the prone position can lead to over-activation of the posterior deltoid, and sub-optimal activation of the infraspinatus contributes to an increase in anterior humeral gliding in the glenoid ${ }^{9,10}$. From this point of view, increased translation of the instantaneous center of rotation of the humeral head may be associated with muscle imbalance between the rotator cuff (e.g., infraspinatus) and the scapulohumeral (e.g., posterior deltoid and middle trapezius) muscles ${ }^{8}$.

Recent shoulder external rotation exercises focus on decreasing the contribution of the posterior deltoid to 
shoulder external rotation to strengthen the infraspinatus muscle isolately ${ }^{2,11}$. Adducted shoulder position isolates the infraspinatus from the posterior deltoid, and side-lying external rotation exercise may be the most effective maneuver to increase infraspinatus activity in isolation ${ }^{5,6}$. To normalize infraspinatus muscle activity among different exercises or positions, it is necessary to isolate the infraspinatus from the surrounding musculature.

Several studies have been conducted to identify methods to normalize the infraspinatus muscle ${ }^{7,12}$. However, there has been no attempt to investigate the optimal MVIC test position for the isolation of the infraspinatus muscle. The objective of this study was to determine the optimal position among four tested positions in which to obtain an isolated infraspinatus EMG signal for the normalization of infraspinatus muscle EMG activity.

\section{Subjects}

The participants in this study were 13 subjects with no known shoulder dysfunction. The mean age, weight, and height of the subjects were $25.2 \pm 3.9$ years, $60.3 \pm 1.2 \mathrm{~kg}$, and $168.4 \pm 5.3 \mathrm{~cm}$, respectively. Before the initial measurements were conducted, each subject completed a health questionnaire and provided informed consent. A screening evaluation of the cervical spine and both upper extremities was performed by a physical therapist.

\section{Instruments}

EMG data were collected using a Noraxon TeleMyo 2400T and analyzed using MyoResearch Master Edition 1.06 XP software (Noraxon Inc., Scottsdale, AZ, USA). Skin preparation of the electrode sites involved shaving and cleaning with rubbing alcohol. Surface electrode pairs were positioned at an interelectrode distance of $2 \mathrm{~cm}$. The reference electrode was placed on the ipsilateral clavicle. EMG data were collected for the following muscles: middle trapezius (midway on a horizontal line between the root of the spine of the scapula and the third thoracic spinous process), upper trapezius (midway between $\mathrm{C} 7$ and the acromion), posterior deltoid (lateral border of the spine of the scapula and angled on an oblique angle toward the arm running parallel to the muscle fiber), and infraspinatus (4 $\mathrm{cm}$ below the spine of the scapula, on the lateral aspect over the infrascapular fossa of the scapula). The sampling rate was $1,000 \mathrm{~Hz}$. The raw signal was full-wave rectified and filtered using a Lancosh FIR digital filter. The band-pass filter frequency was between 20 and $300 \mathrm{~Hz}$. EMG data were converted to root mean square (RMS) values, which were calculated from $50 \mathrm{~ms}$ windows within the data points.

\section{Procedure}

The dominant arm, defined as the upper extremity used to eat and write, was tested for all participants. All participants were right-arm dominant, as determined by a questionnaire. A target bar was placed such that the radial border of the participant's wrist touched it in each test position. To measure MVIC in the side-lying with the shoulder adducted (ADD) position, subjects lay on their sides with the shoulder in a neutral position and the elbow at $90^{\circ}$ flexion. From the starting position, with the forearm resting on the abdomen, subjects moved the tested arm to a position of external rotation $\left(45^{\circ}\right)$ until the dorsal forearm touched the target bar and maintained the shoulder at $45^{\circ}$ external rotation $^{6}$. The subject then performed maximal contraction of the dominant side arm by applying manual resistance to pressure by an examiner to the subject's wrist. All subjects were given consistent verbal encouragement during maximal contraction. In the prone position, the subject was required to lie prone with the shoulder abducted at $90^{\circ}$ and the elbow flexed to $90^{\circ}$, while the forearm was in neutral position ${ }^{5}$. Additionally, the subject's elbow was fastened to the table using a non-elastic belt to prevent compensatory shoulder motion. To measure MVIC in the prone position, the subject moved to a position with shoulder external rotation to $90^{\circ}$. The subject then performed maximal contraction of the dominant side arm by applying manual resistance to pressure by an examiner to the subject's wrist. All subjects were given consistent verbal encouragement during maximal contraction. To measure MVIC in shoulder abducted (ABD) position, the participant stood with the dominant arm abducted and internally rotated to $90^{\circ}$ and the elbow flexed to $90^{\circ}$. From the starting position, the participant moved the extremity to a position of full lateral rotation until the wrist joint touched the target bar. The subject then performed maximal contraction of the dominant side arm by applying manual resistance to pressure by an examiner to the subject's wrist. All subjects were given consistent verbal encouragement during maximal contraction. To measure MVIC in the side-lying wiper external rotation (SWE) position, the participant lay in the side-lying position with the extremity flexed and internally rotated to $90^{\circ}$ and the elbow flexed to $90^{\circ}$. The participant supported the distal humerus of the dominant arm with the palm of the opposite hand. From the starting position, the participant moved to a position of full lateral rotation until the wrist joint touched the target bar. We instructed the participant to use the opposite hand to support the dominant arm during external rotation to maintain the center of rotation in the same position. 
The familiarization period was completed when the subject was able to maintain three normalization methods for $5 \mathrm{~s}$. All subjects were comfortable after the familiarization period, and none reported fatigue. A 15-min rest period was allowed after the familiarization period before data collection began. The order of testing was randomized using a random number generator (Microsoft Corp., Redmond, WA, USA).

EMG activity was measured during each normalization trial for $5 \mathrm{~s}$. The first and last seconds of the EMG data from each trial were discarded, and the remaining $3 \mathrm{~s}$ of data were used for further analysis. Subjects performed the second session $1 \mathrm{~h}$ after the first session, following the identical protocol. Three trials were performed with a 1-min rest period between trials. A 3-min rest period was provided between conditions to minimize muscle fatigue.

\section{Statistical analysis}

The Shapiro-Wilks test was performed to verify the normality of the data distribution. A repeated one-way analysis of variance was performed to determine the muscle activity ratio among four test positions. The Bonferroni procedure was used as a post-hoc analysis. The level of significance was set at $p<0.05$. Significant differences were evaluated using the Bonferroni adjustment at a level of $0.013(0.05 / 4)$. All statistical tests were performed using SPSS v. 23.0 software (SPSS, Inc., Chicago, IL, USA).

\section{RESULTS}

The EMG ratio $\left(100 \times \frac{\text { infraspinatus muslce activity }}{\text { middle trapezius }}\right.$ infraspinatus muslce activity $\frac{\text { upper trapezius+posterior deltoid muscle activities }}{\text { ) and }}$ results of the statistical analyses are shown in Table 1 $(\mathrm{F}=24.96, p<0.00)$. The EMG ratio was significantly greater when the subject was in the SWE position than in all other test positions $(p<0.05)$, following the order SWE $>$ ADD $>$ PRE $>$ ABD.

\section{DISCUSSION}

The objective of this study was to determine the best position in which to obtain an isolated infraspinatus EMG signal that could be used to normalize the infraspinatus
EMG activity among four selected positions. Kelly et al. (1996) reported that standardizing normalization test positions for MVIC is one step toward the elimination of external variation ${ }^{13}$. Specifically, compensatory movement or muscle activation in the surrounding muscles leads to decreasing reliability of the normalization of the infraspinatus muscle.

Our study shows that the EMG ratio was significantly higher in the SWE position than in all other test positions. To maximize the EMG ratio, high infraspinatus activity and low surrounding muscle (middle trapezius, upper trapezius, and posterior deltoid) activity are required. Our results are consistent with those of several previous studies, which reported that SWE is an effective exercise position to isolate the activity of the infraspinatus muscle while minimizing surrounding muscle activity $6,11,14$. There are several possible explanations for our results. First, infraspinatus muscle activation is more dependent on arm and body position than that of the surrounding muscles ${ }^{11}$. Previous studies have reported that maximum infraspinatus muscle activity occurred during shoulder forward flexion at $90^{\circ}$. In this study, SWE was performed with the shoulder flexed to $90^{\circ}$, whereas the other exercises were performed with the shoulder abducted to $90^{\circ}$ or $0^{\circ}$. Second, SWE allowed the subject to maintain the instantaneous center of rotation of the humeral head. Shoulder external rotation is often accompanied by unnecessary shoulder movement. In the SWE position, manual fixation of the humerus prevented unnecessary movement, which induced increased infraspinatus muscle activity while decreasing surrounding muscle activity. The other normalization positions led to an over-activated posterior deltoid and a less-activated infraspinatus (i.e., a low EMG ratio). Hyper-activation of the surrounding muscle (middle trapezius and posterior deltoid) often induces abnormal gleno-humeral and scapula-thoracic joint motion, which consequently increases the instability of the shoulder joint ${ }^{9,10}$. When the posterior deltoid is the dominant external rotator, unwanted anterior glide of the humeral head during shoulder external rotation can result. Based on the results of our study, we recommend a switch from prone external rotation to SWE for the normalization of infraspinatus EMG activity.

This study has some limitations. The generalizability of the study is limited because we recruited only young

Table 1. Comparison for EMG ratio among 4 different manual muscle testing position

$(\mathrm{n}=19)$

\begin{tabular}{ccccccc}
\hline & PRE & SWE & ABD & ADD & $F$ & $p$ \\
\hline EMG ratio & $28.72 \pm 6.33$ & $77.46 \pm 34.20$ & $20.68 \pm 3.52$ & $33.15 \pm 14.69$ & 24.96 & $<0.00$ \\
\hline
\end{tabular}

PRE, prone external rotation; SWE, side-lying wiper exercise; ABD, shoulder abducted; ADD, shoulder adducted. 
Table 2. Multiple comparison between tests.

\begin{tabular}{cl}
\hline Test comparison & $p$ \\
\hline PRE versus SWE & $0.00^{*}$ \\
\hline PRE versus ABD & $0.01^{*}$ \\
PRE versus ADD & 1.00 \\
SWE versus ABD & $0.00^{*}$ \\
SWE versus ADD & $0.00^{*}$ \\
ABD versus ADD & 0.03 \\
\hline
\end{tabular}

PRE, prone external rotation; SWE, side-lying wiper exercise; ABD, shoulder abducted; ADD, shoulder adducted.

healthy subjects; future studies should determine whether our findings can be generalized to subjects beyond this age range. We also did not measure force or torque during normalization measurements; force and torque should be examined in future studies. Multiple comparisons between positions are presented in Table 2.

\section{CONCLUSION}

We investigated the EMG ratio of the infraspinatus muscles and surrounding muscle (middle trapezius and posterior deltoid) during normalization of the infraspinatus muscle in four selected positions. Our findings show that the EMG ratio was significantly higher in the SWE position than in all other tested positions. To maximize the EMG ratio, high infraspinatus activity and low surrounding muscle (middle trapezius and posterior deltoid) activity are required. Our results are consistent with those of several previous studies, which reported that SWE is an effective exercise position to isolate the activity of the infraspinatus muscle while minimizing surrounding muscle activity. These findings suggest that SWE is more effective than other positions for the normalization of infraspinatus EMG activity.

\section{Key Points}

Question Which position isolatable activate the infraspinatus muscle?

Findings Our study showed that the electromyography (EMG) ratio was significantly higher and side-lying external rotation (SWE) than other test position. For the highest EMG ratio, it necessary high infraspinatus and low surrounding muscle (middle trapezius and posterior deltoid) activities. Consistent with our results, several studies have been reported that SWE is effective exercise position for isolate activating the infraspinatus muscle while minimizing sur- rounding muscle activation.

Meaning Based on the results of our study, it seems necessary to change the normalization for infraspinatus EMG from PRE (prone external rotation) to SWE.

\section{Article information}

Conflict of Interest Disclosures: None.

Funding/Support: None.

Acknowledgment: None.

\section{REFERENCES}

1. Ballantyne BT, O'Hare SJ, Paschall JL, Pavia-Smith MM, Pitz AM, Gillon JF, Soderberg GL. Electromyographic activity of selected shoulder muscles in commonly used therapeutic exercises. Phys Ther. 1993;73(10):668-677.

2. Bitter NL, Clisby EF, Jones MA, Magarey ME, Jaberzadeh S, Sandow MJ. Relative contributions of infraspinatus and deltoid during external rotation in healthy shoulders. J Shoulder Elbow Surg. 2007;16(5):563568.

3. Ekstrom RA, Donatelli RA, Soderberg GL. Surface electromyographic analysis of exercises for the trapezius and serratus anterior muscles. J Orthop Sports Phys Ther. 2000;33(5):247-258.

4. Allison GT, Godfrey P, Robinson G. EMG signal amplitude assessment during abdominal bracing and hollowing. J Electromyogr Kinesiol. 1998;8(1):51-57.

5. Reinold MM, Wilk KE, Fleisig GS, Zheng N, Barrentine SW, Chmielewski T, Cody RC, Jameson GG, Andrews JR. Electromyographic analysis of the rotator cuff and deltoid musculature during common shoulder external rotation exercises. J Orthop Sports Phys Ther. 2004; 34(7):385-394.

6. Ha SM, Kwon OY, Cynn HS, Lee WH, Kim SJ, Park KN. Selective activation of the infraspinatus muscle. $J$ Athl Train. 2013;48(3):346-352.

7. Ha SM, Cynn HS, Kwon OY, Park KN, Kim GM. A reliability of electromyographic normalization methods for the infraspinatus muscle in healthy subjects. J Hum Kinet. 2013;28(36):69-76.

8. Kang MH, Oh JS, Jang JH. Differences in muscle activities of the infraspinatus and posterior deltoid during shoulder external rotation in open kinetic chain and closed kinetic chain exercises. J Phys Ther Sci. 2014; 26(6):895-897.

9. Caldwell C, Sahrmann S, Van Dillen L. Use of a movement system impairment diagnosis for physical therapy in the management of a patient with shoulder pain. $J$ 
Orthop Sports Phys Ther. 2007;37(9):551-563.

10. Sharmann S. Diagnosis and treatment of movement impairment syndromes. 1st ed. St. Louis: Mosby; 2002.

11. Kim JW, Yoon JY, Kang MH, et al. Selective activation of the infraspinatus during various shoulder external rotation exercises. J Phys Ther Sci. 2012;24(7):581-584.

12. Alenabi T, Whittaker R, Kim SY, Dickerson CR. Maximal voluntary isometric contraction tests for normalizing electromyographic data from different regions of supraspinatus and infraspinatus muscles: Identifying reliable combinations. J Electromyogr Kinesiol. 2018;
41:19-26

13. Kelly BT, Kadrmas WR, Kirkendall DT, Speer KP. Optimal normalization tests for shoulder muscle activation: an electromyographic study. J Orthop Res. 1996;14(4): 647-53.

14. Kim HA, Hwang UJ, Jung SH, Ahn SH, Kim JH, Kwon OY. Effect of horizontal adduction force on infraspinatus and deltoid activities during the side-lying wiper exercise using pressure biofeedback. Physical Therapy Korea. 2017;24(4):77-83. 